\title{
A Universal Condition Satisfied by the Action of Electromagnetic Radiation Fields
}

\author{
Vernon Cooray ${ }^{1}$, Gerald Cooray ${ }^{2}$ \\ ${ }^{1}$ Department of Engineering Sciences, Uppsala University, Uppsala, Sweden \\ ${ }^{2}$ Karolinska Institute, Stockholm, Sweden \\ Email:Vernon.Cooray@angstrom.uu.se
}

How to cite this paper: Cooray, V. and Cooray, G. (2017) A Universal Condition Satisfied by the Action of Electromagnetic Radiation Fields. Journal of Electromagnetic Analysis and Applications, 9, 167-182. https://doi.org/10.4236/jemaa.2017.911015

Received: October 6, 2017

Accepted: November 27, 2017

Published: November 30, 2017

Copyright ( 92017 by authors and Scientific Research Publishing Inc. This work is licensed under the Creative Commons Attribution International License (CC BY 4.0).

http://creativecommons.org/licenses/by/4.0/

(c) (i) Open Access

\begin{abstract}
The action (the product of radiated energy and the time of emission) of the radiation fields generated by four types of radiators, namely, short electric dipole, small magnetic dipole, travelling wave antenna and bi-conical antenna is investigated with special reference to the charge associated with the current waveform which is responsible for the radiation. The results obtained can be summarized by the order of magnitude inequality $A \geq h / 2 \pi \rightarrow q \geq e$ where $A$ is the action (product of the radiated energy and the time of emission), $h$ is the Planck constant, $q$ is the charge associated with the current that gave rise to the radiation and $e$ is the electronic charge. The condition $A \approx h / 2 \pi \rightarrow q \approx e$ is obtained when the length of the antenna and its radius are pushed to its extreme natural limits. Based on the results obtained here and elsewhere, it is suggested that this inequality is valid in general for electromagnetic radiation fields as predicted by classical electrodynamics.
\end{abstract}

\section{Keywords}

Classical Electrodynamics, Electromagnetic Radiation, The Action of Electromagnetic Radiation, Elementary Charge, Electronic Charge, Time-Energy Uncertainty Principle

\section{Introduction}

Electromagnetic fields generated by transmitting antennas can be calculated once the current and its propagation characteristics along the antenna are known. In the case of short electric dipole and small magnetic dipole, one needs only the information concerning the temporal variation of the current waveform and the length of the electric dipole or the area of the magnetic dipole to evaluate the electromagnetic fields. As the dipole length increases it is necessary to 
include the time delays caused by the propagation of the current along the antenna in calculating the electromagnetic fields. Such antennas can be categorized as travelling wave antennas. In an ideal travelling wave antenna, a current pulse propagates from one end of the antenna to the other end with the speed of light without attenuation or dispersion. The scenario is somewhat similar to the transmission line model of lightning return strokes with a speed of light current propagation [1]. Another type of transmitting antenna used frequently is the bi-conical antenna. Such an antenna consists of a conducting cone located above a conducting half space. Current waveforms propagate along such an antenna with the speed of light in free space. Actually, in some studies, the lightning return stroke is also described as a limiting case of a bi-conical antenna where the semi-conical angle approaches zero [2].

Once the wave shape of the current and its propagation characteristics are known, the standard techniques based on classical electrodynamics can be used to analyze the features of radiation fields such as the energy, momentum and the action transported by these fields. The action of a radiation field is defined here as the product of the energy emitted by the radiation and the corresponding time of emission.

In practical applications related to the transmission of electromagnetic signals or in the case of lightning, one is interested in current waveforms carrying charges on the order of milli-Coulombs to several Coulombs. For this reason, no one has investigated the nature of the lower limits of the energy and the action of the radiation fields as the charge associated with the current in the radiating antenna is reduced to the smallest values allowed by nature. In a recent study Cooray and Cooray [3] [4] [5] investigated the nature of the radiation fields, especially their action, as the charge associated with the radiating element is reduced to the elementary charge. They found that, for an electromagnetic radiator confined by natural bounds, the action of the radiation field satisfies the inequality $A \geq h / 2 \pi \rightarrow q \geq e$ where $A$ is the action associated with the radiation field, $h$ is the Planck constant, $q$ is the charge associated with the current and $e$ is the electronic charge. This was shown to be valid also for the radiation produced by accelerating or decelerating electrons as they strike a perfectly conducting plane in a recent publication by Cooray and Cooray [6]. Even though the Planck constant appears in the expression, the calculation is based purely on classical electrodynamics. It is important to mention here that the charge of an electron is not something special in classical electrodynamics. The fact that the electric charge is quantized and the smallest charge that can occur in nature is the elementary charge was discovered nearly 35 years after the development of classical electrodynamics. In this paper, the validity of the inequality given earlier is established for the radiation fields emitted by short electric dipoles, small magnetic dipoles, travelling wave antennas and bi-conical antennas.

\section{Current Waveform Used in the Analysis}

In the analysis, the current waveform associated with the antenna is represented 
by a Gaussian pulse. It can be described mathematically as

$$
i(t)=i_{0} \mathrm{e}^{-t^{2} / 2 \sigma^{2}}
$$

In the above equation $i_{0}$ is a constant having units of Amperes, $t$ is the time and $\sigma$ is a parameter that controls the width of the Gaussian current pulse. The charge, $q$, associated with this current pulse is given by

$$
q=i_{0} \tau
$$

In Equation (2), $\tau$ is the duration of the current pulse and it is given by

$$
\tau=\sqrt{2 \pi \sigma^{2}}
$$

Thus, the current pulse can also be written as

$$
i(t)=\frac{q}{\sqrt{2 \pi \sigma^{2}}} \mathrm{e}^{-t^{2} / 2 \sigma^{2}}
$$

In order to make the analysis general, let us define a non-dimensional parameter $\beta$ as

$$
\beta=\tau /(l / c)
$$

where $l$ is the length of the antenna and $c$ is the speed of light (later, we will define $\beta$ in a different way for a magnetic dipole). Observe that $\beta$ defines the ratio between the duration of the current and the time of travel of the current along the antenna.

\section{Analysis}

In the analysis to follow we will consider four types of time domain radiators namely, short electric dipole, small magnetic dipole, travelling wave antenna and bi-conical antenna.

\subsection{Short Electric Dipole}

A classic example of an electromagnetic radiating system is the short electric dipole (or Hertzian electric dipole). A unique aspect of the short electric dipole is that most of the complex and spatially extended radiating systems can be described as a distribution of short electric dipoles. The radiation field generated by the complex system can be described, in this case, as the sum of radiation fields generated by the collection of short dipoles. In this analysis we will consider a short electric dipole of length $l$ excited by a transient current. In a short electric dipole $l / c \ll \tau$ where, $\tau$ as denoted previously, is the duration of the current. That is for a short dipole $\beta \gg 1$.

\subsubsection{Electromagnetic Fields of a Short Electric Dipole}

The electric field produced by a short electric dipole consists of three terms, namely, electrostatic, induction and radiation [7]. Since our goal is to study the energy radiated by the dipole it is sufficient to concentrate only on the radiation field terms. Let us consider a short dipole excited by a current which is denoted 
here by $i(t)$ where $t$ is the time. The electric radiation field produced by a short dipole of length $l$ in free space is given by (see Figure 1 for the relevant geometry) [7]:

$$
\boldsymbol{E}_{\theta}=\frac{l \sin \theta}{4 \pi \varepsilon_{0} c^{2} r} \frac{\mathrm{di}(t-r / c)}{\mathrm{d} t} \boldsymbol{a}_{\theta}
$$

Note that $\boldsymbol{a}_{\theta}$ and $\boldsymbol{a}_{\phi}$ are unit vectors in the increasing $\theta$ and $\phi$ directions. This equation is valid provided that $\beta \gg 1$ and $r \gg 1$. The magnetic flux density (or the B-field) associated with the radiation is directed along $\boldsymbol{a}_{\phi}$ and has the magnitude $E_{\theta} / c$. The Poynting vector associated with the radiation field is directed in the radial direction and it is given by

$$
\boldsymbol{S}(\theta, t)=\left(\frac{l \sin \theta}{4 \pi c r}\right)^{2} \frac{1}{\varepsilon_{0} c}\left[\frac{\mathrm{di}(t-r / c)}{\mathrm{d} t}\right]^{2} \boldsymbol{a}_{r}
$$

The power radiated by the dipole as a function of time can be obtained by integrating the Poynting vector over a spherical surface centered at the dipole. The result is

$$
P(t)=\frac{l^{2}}{6 \pi \varepsilon c^{3}}\left[\frac{\mathrm{d} i(t-r / c)}{\mathrm{d} t}\right]^{2}
$$

\subsubsection{Energy Dissipation and the Action Associated with the Radiation of a Short Dipole}

The total energy radiated by the dipole, $U$, can be obtained by integrating the power given by Equation (8) over time. The result, based on the current waveform given in Equation (4), is

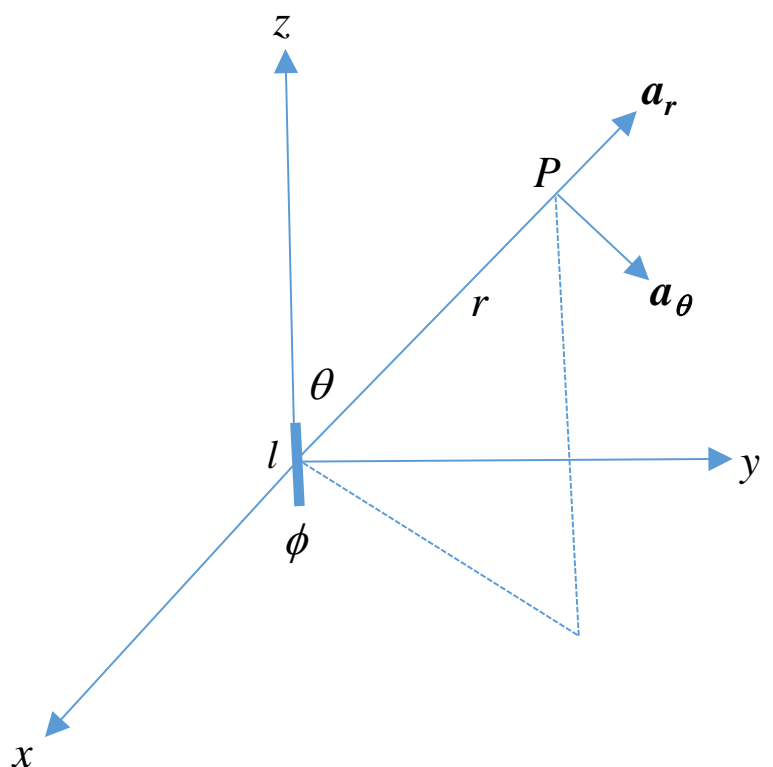

Figure 1. The geometry relevant to the description of electromagnetic fields of a short electric dipole of length $I$ located at the origin of the axes. In the diagram $\boldsymbol{a}_{r}$ and $\boldsymbol{a}_{\boldsymbol{\theta}}$ are unit vectors in the direction of increasing $r$ and $\theta$ respectively. The unit vector $\boldsymbol{a}_{\phi}$ is directed along the vector $\boldsymbol{a}_{r} \times \boldsymbol{a}_{\theta}$. 


$$
U=\frac{q^{2} l^{2} \sqrt{2 \pi}}{12 \pi^{2} \varepsilon_{0}} \frac{1}{c^{3} \sigma^{3}}
$$

Substituting for $\sigma$ in terms of $\tau$ from Equation (3) we obtain

$$
U=\frac{q^{2} l^{2}}{3 \varepsilon_{0}} \frac{1}{c^{3} \tau^{3}}
$$

The action associated with the radiation field is then given by

$$
A=\tau U=\frac{q^{2}}{3 \varepsilon_{0} c}\left[\frac{1}{\beta}\right]^{2}
$$

Now, as mentioned previously, in the case of a short dipole $t \gg 1 / c$, i.e. $\beta \gg 1$. Thus the upper limit to the action associated with the radiation produced by a short dipole, $A_{m}$, depends on the smallest possible value of $\beta$. Since $\beta \gg 1$, the smallest possible value of $\beta$, still within the dipole limit, is about 3 . Since the action increases with decreasing value of $\beta$ an absolute upper ceiling for the action can be obtained by substituting $\beta=1$ in Equation (11). That is,

$$
A_{m}=\frac{q^{2}}{3 \varepsilon_{0} c}
$$

Figure 2 shows how this action varies as a function of charge. In the diagram the action is given as a fraction of $h / 2 \pi$ where $h$ is the Planck constant and the charge is given as a fraction of the elementary charge $e$. Note that the charge necessary to reach one unit of action is about $6 e$. This shows that the short electric dipole radiation satisfies the inequality $A>h / 2 \pi \rightarrow q>e$.

\subsection{Small Magnetic Dipole}

A magnetic dipole consists of a closed loop around which an electric current is

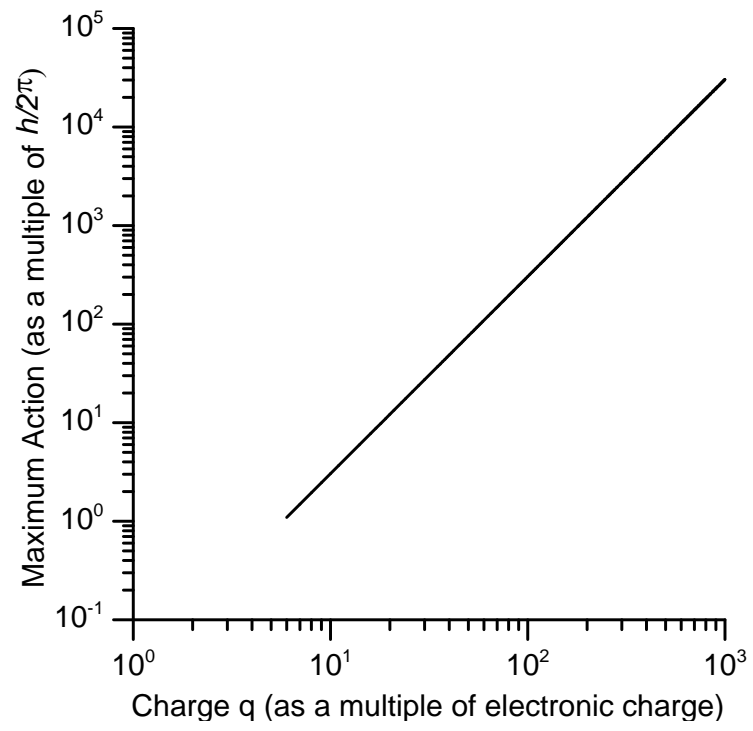

Figure 2. The maximum action (in multiples of $h / 2 \pi$ ) as a function of the charge $q$ (in multiples of the electronic charge) pertinent to a short dipole. Note that the diagram corresponds to the case with the limiting value of $\beta=1$. 
flowing. In this analysis we will consider a small loop of radius $a$ excited by a transient current. In the case of a magnetic dipole $2 \pi a / c \ll \tau$ where, $\tau$ as denoted previously, is the duration of the current. That is, the time for the current to propagate around the loop is much smaller than the duration of the current. In order to make the analysis very general let us define a parameter $\beta_{m}$ as follows:

$$
\tau=\beta_{m} 2 \pi a / c
$$

Thus, for a magnetic dipole $\beta_{m} \gg 1$.

\subsubsection{Electromagnetic Fields of a Small Magnetic Dipole}

Since our goal is to study the energy radiated by the magnetic dipole it is sufficient to concentrate only on the radiation field terms. The geometry relevant to the field calculation is given in Figure 3. The electric radiation field produced by a magnetic dipole of radius $a$ in free space is given by [7]:

$$
\boldsymbol{E}_{\phi}=-\frac{\mu_{0} A \sin \theta}{4 \pi \varepsilon_{0} c r} \frac{\operatorname{di}(t-r / c)}{\mathrm{d} t} \boldsymbol{a}_{\phi}
$$

In the above equation $A$ is the area of the magnetic dipole. This equation is valid provided that $\beta_{m} \gg 1$ and $r \gg a$. The magnetic flux density (or the B-field) associated with the radiation is directed along $\boldsymbol{a}_{\boldsymbol{\theta}}$ and has the magnitude $E_{\phi} / c$. The Poynting vector associated with the radiation field is directed in the radial direction and it is given by

$$
\boldsymbol{S}(\theta, t)=\left(\frac{A \sin \theta}{4 \pi r}\right)^{2} \frac{\mu_{0}}{c^{3}}\left[\frac{\mathrm{d}^{2} i(t-r / c)}{\mathrm{d} t^{2}}\right]^{2} \boldsymbol{a}_{r}
$$

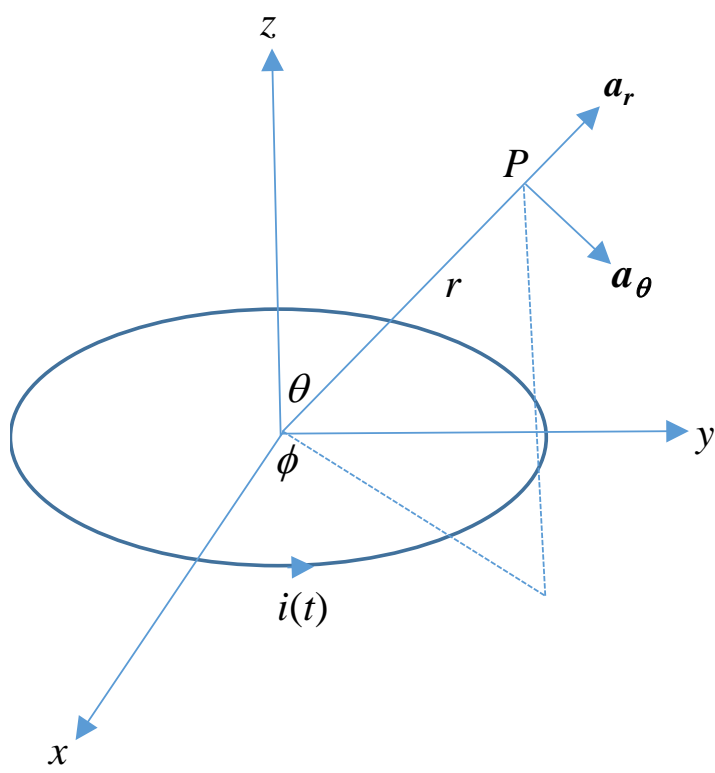

Figure 3. The geometry relevant to the description of electromagnetic fields of a small magnetic dipole of length radius $a$ located at the origin of the axes. In the diagram $\boldsymbol{a}_{r}$ and $\boldsymbol{a}_{\boldsymbol{\theta}}$ are the unit vectors in the direction of increasing $r$ and $\theta$. The unit vector $\boldsymbol{a}_{\phi}$ is directed along the vector $\boldsymbol{a}_{r} \times \boldsymbol{a}_{\theta}$. 
The power radiated by the magnetic dipole as a function of time can be obtained by integrating the Poynting vector over a spherical surface centered at the dipole. The result is

$$
P(t)=\frac{\mu_{0} A^{2}}{6 \pi c^{3}}\left[\frac{\mathrm{d}^{2} i(t-r / c)}{\mathrm{d} t^{2}}\right]^{2}
$$

\subsubsection{Energy Dissipation and the Action Associated with the Radiation of a Small Magnetic Dipole}

The total energy radiated by the magnetic dipole, $U$, can be obtained by integrating the power given by Equation (16) over time. The result, based on the current waveform given in Equation (4), is

$$
U=\frac{\mu_{0} q^{2} A^{2}}{16 \pi^{3 / 2} c^{3}} \frac{1}{\sigma^{5}}
$$

Substituting for $\sigma$ in terms of $\tau$ from Equation (3), the energy dissipated as radiation can be expressed as

$$
U=\frac{\mu_{0} q^{2}}{64 \pi^{3 / 2}} \frac{c}{\sigma}\left(\frac{1}{\beta_{m}}\right)^{4}
$$

The action associated with the radiation field is then given by

$$
A=\tau U=\frac{q^{2}}{32 \pi \varepsilon_{0} c \sqrt{2}}\left(\frac{1}{\beta_{m}}\right)^{4}
$$

Now, as mentioned previously, in the case of a small magnetic dipole $\tau \gg 2 \pi a / c$, i.e. $\beta_{m} \gg 1$. Thus the upper limit to the action associated with the radiation produced by a small dipole, $A_{m}$, depends on the smallest possible value of $\beta_{m}$. Since $\beta_{m} \gg 1$, the smallest possible value of $\beta_{m}$, still within the dipole limit, is about 3. Since the action increases with decreasing value of $\beta_{m}$, as in the case of the electric dipole, an absolute upper ceiling for the action can be obtained by substituting $\beta_{m}=1$ in Equation (19). That is,

$$
A_{m}=\frac{q^{2}}{32 \pi \varepsilon_{0} c \sqrt{2}}
$$

Figure 4 shows how this action varies as a function of charge. In the diagram the action is given as a fraction of $h / 2 \pi$ and the charge is given as a fraction of the elementary charge. Note that the charge necessary to reach one unit of action is about 38e. This is much larger than the value we obtained for the electric dipole. In other words, for a given charge the electric dipole is a much more efficient radiator than a magnetic dipole. However, the magnetic dipole radiation still satisfies the inequality $A>h / 2 \pi \rightarrow q>e$.

\subsection{Travelling Wave Antenna}

In an ideal travelling wave antenna, a current pulse propagates from one end of a conductor to the other end with the speed of light and without attenuation [8]. The current is assumed not to be reflected at the point of termination. Indeed, 


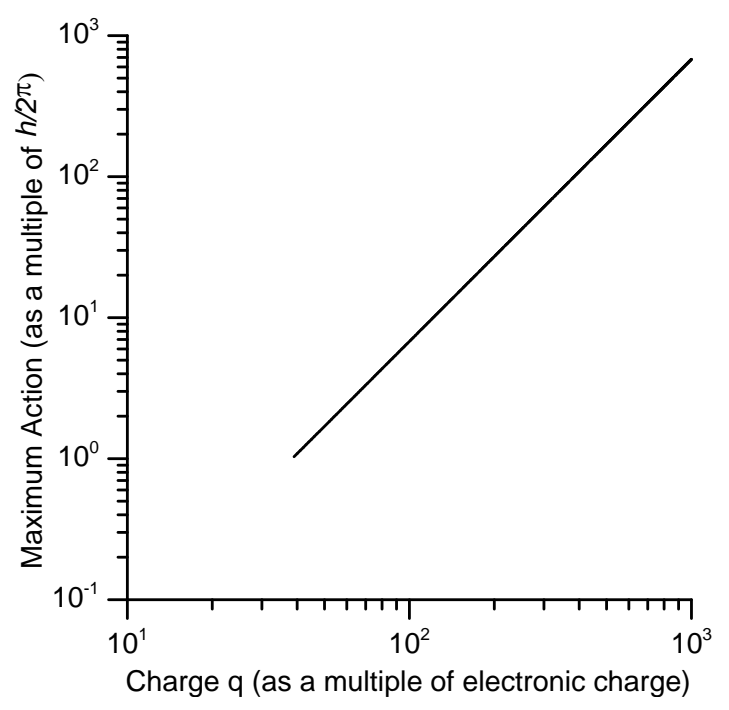

Figure 4. The maximum action (in multiples of $h / 2 \pi$ ) as a function of the charge $q$ (in multiples of the electronic charge) pertinent to a small magnetic dipole. Note that the diagram corresponds to the case with the limiting value of $\beta_{m}=1$.

this scenario is valid for an ideal antenna where all the losses are neglected. As mentioned earlier, this physical picture is identical to that utilized in the transmission line model used to represent lightning return strokes when the speed of propagation of the return stroke front is equal to the speed of light [1] [2].

\subsubsection{Electromagnetic Fields of a Travelling Wave Antenna}

The geometry relevant to the derivation of the electromagnetic field equations is shown in Figure 5. A current pulse is initiated at $S_{1}$ and propagates toward $S_{2}$ with the speed of light without attenuation or dispersion. The current is terminated at $S_{2}$. The system will generate two radiation field pulses one at the initiation of the current and another one during the termination of the current. Let us consider a point $P$ which satisfies the condition $r \gg l$ and where only the radiation fields exist. When point $P$ is located far away from the antenna the three lines connecting $P$ in Figure 5 are essentially parallel and that allows us to write,

$$
r_{1}=r-l \cos \theta
$$

Let us also define the parameters $\delta$ as

$$
\delta=l(1-\cos \theta) / c
$$

The electric radiation field at the point of observation is given for $r / c<t<r / c+\delta$ (reader is referred to [7] for the details of this derivation)

$$
\boldsymbol{E}_{\theta}=\frac{\sin \theta}{4 \pi \varepsilon_{o} c r} \frac{i(t-r / c)}{[1-\cos \theta]} \boldsymbol{a}_{\theta}
$$

and for $t>r / c+\delta$ the field is given by

$$
\boldsymbol{E}_{\theta}=\frac{\sin \theta}{4 \pi \varepsilon_{0} c r[1-\cos \theta]}(i(t-r / c)-i(t-r / c-\delta)) \boldsymbol{a}_{\theta}
$$




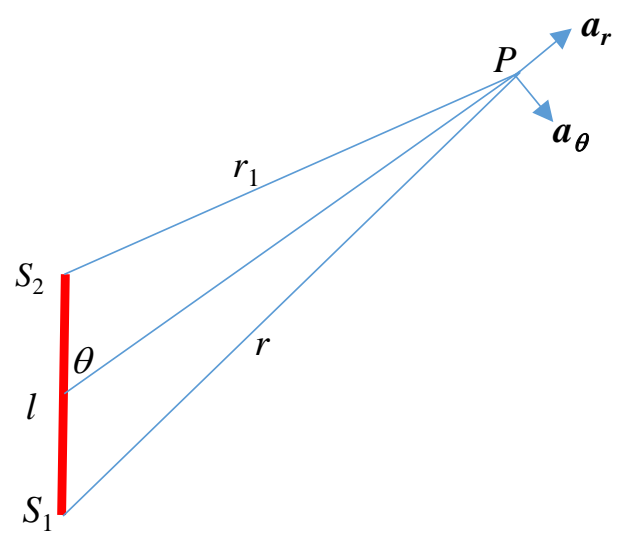

Figure 5. Geometry relevant to the derivation of field equations pertinent to a travelling wave antenna. The unit vector $\boldsymbol{a}_{\phi}$ is directed along the vector $\boldsymbol{a}_{r} \times \boldsymbol{a}_{\theta}$.

The radiation field consists of two bursts, one generated during the initiation of the current pulse at $S_{1}$ and the other during the termination of the current pulse at $S_{2}$. The duration of the radiation field pulses generated during the current initiation and current termination at the two ends of the conductor is equal to the duration of the current. The magnetic flux density (or the B-field) associated with the radiation is directed along $\boldsymbol{a}_{\phi}$ and has the magnitude $E_{\theta} / c$. The Poynting vector associated with these fields is given, for $r / c<t<r / c+\delta$, by

$$
\boldsymbol{S}(\theta, t)=\frac{i(t-r / c)^{2} \sin ^{2} \theta}{(4 \pi)^{2} \varepsilon_{0} c r^{2}} \frac{1}{[1-\cos \theta]^{2}} \boldsymbol{a}_{r}
$$

and for $t>r / c+\delta$

$$
\boldsymbol{S}(\theta, t)=\frac{\sin ^{2} \theta}{(4 \pi)^{2} \varepsilon_{0} c r^{2}} \frac{[i(t-r / c)-i(t-r / c-\delta)]^{2}}{[1-\cos \theta]^{2}} \boldsymbol{a}_{r}
$$

The power dissipated by the radiation field is then given by

$$
P(t)=\int_{0}^{2 \pi} \int_{0}^{\pi} S(\theta, t) r^{2} \sin \theta \mathrm{d} \theta \mathrm{d} \phi
$$

The net energy dissipated by the radiation field can be obtained by integrating the above expression over time.

\subsubsection{Energy Dissipation and the Action Associated with the Radiation of the Travelling Wave Antenna}

The total energy radiated, $\Delta U$, by the travelling wave antenna can be calculated numerically for any given value of $\beta$. However, Cooray and Cooray [4] showed that the cumbersome expression for the energy dissipation reduces to a simple expression when $\beta<10^{-6}$. That expression is given by (this can be obtained by performing the integral in Equation (27) of [4])

$$
\Delta U=\frac{q^{2}}{4 \pi^{3 / 2} \varepsilon_{0} c \sigma} \ln \frac{1}{\beta}
$$


Note that the energy emitted by the radiation for a given $\beta$ is both a function of charge and $\sigma$. The action associated with the radiation field, $A$, is given by

$$
A=\Delta U \tau
$$

Substituting for $\Delta U$ from Equation (28) we obtain

$$
A=\frac{\tau q^{2}}{4 \pi^{3 / 2} \varepsilon_{0} c \sigma} \ln \frac{1}{\beta}
$$

Substituting for $\tau$ from Equation (3) and noting that $\beta=l / c \tau$ we obtain

$$
A=\frac{\sqrt{2} q^{2}}{4 \pi \varepsilon_{0} c} \ln \frac{l}{c \tau}
$$

In writing down the expression for the radiation fields we have not considered the effect of the radius of the conductor. However, we have tacitly assumed that the radiation generated from any point across the cross section of the antenna is in phase. This is strictly true only when the duration of the current waveform is larger than the time $a / c$ where $a$ is the radius of the conductor. Thus, the smallest value of $\tau$ that one can use in the equation is on the order of $a / c$ and when this is substituted in Equation (31) we obtain

$$
A=\frac{\sqrt{2} q^{2}}{4 \pi \varepsilon_{0} c} \ln \frac{l}{a}
$$

In Equation (32) we have the final expression necessary to study how the upper limit of the action of the radiation field varies as a function of $q$, $a$ and $l$. Observe from Equation (32) that the action associated with a given charge increases with increasing $l$ and with decreasing $a$. The radius of a conductor or a conducting channel in nature cannot be smaller than the atomic dimensions or the Bohr radius, $a_{0}$. Thus, for a given $q$ and $l$ the maximum action is obtained for $a=$ $a_{0}$. Note also that the action increases with increasing $l$. The maximum value of $l$ that can be achieved in nature cannot be larger than the radius of the universe, $R_{u}$. For this reason, the maximum action that can be obtained for a travelling wave antenna excited by a given charge $q$ is given by

$$
A_{m}=\frac{\sqrt{2} q^{2}}{4 \pi \varepsilon_{0} c} \ln \frac{R_{u}}{a_{0}}
$$

This action (given in units of $h / 2 \pi$ ) as a function of charge $q$ (given in units of electronic charge) is shown in Figure 6. In making this diagram we have taken $a_{0}=5.29 \times 10^{-11} \mathrm{~m}$ and $R_{u}=4.4 \times 10^{26} \mathrm{~m}$ (one can of course use either the size of the observable universe or the current Hubble radius but in either case the order of magnitude of the result remains the same). Observe that as the charge reduces to the electronic charge, the maximum action reduces to a value on the order of $h / 2 \pi$. This shows that, within the confines of the natural limits, the maximum action of the radiation field of a travelling wave antenna excited by an electronic charge is about $h / 2 \pi$. If the charge is reduced below this critical value the action will also be reduced below $h / 2 \pi$. We can summarize this result using the inequality $A \geq h / 2 \pi \rightarrow q \geq e$. 


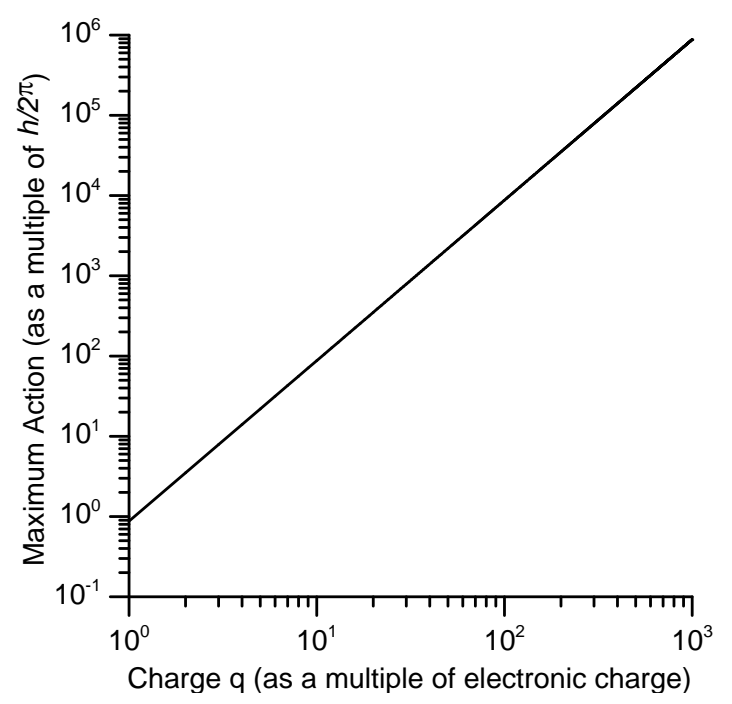

Figure 6. The maximum action (in multiples of $h / 2 \pi$ ) as a function of the charge $q$ (in multiples of electronic charge) pertinent to a wave antenna. Note that as $q$ reaches the electronic charge the maximum action reaches about $h / 2 \pi$.

Observe that if we consider a smaller antenna length the action becomes smaller and a larger charge will be needed to maintain the action above $h / 2 \pi$. The same is true if we increase the duration of the current while keeping the antenna length constant. For a given antenna length, as the duration of the current increases the action decreases and again one needs a larger charge to reach the critical action. This is also true if we try to increase the radius of the conductor while keeping the duration of the current constant. As the radius increases a stage will be reached at which the radiation from different parts of the cross section of the current path interfere destructively reducing the action. The case is the same if we take into account any attenuation of the current waveform along the channel. This will reduce the action leaving the inequality given above unaffected.

\subsection{Bi-Conical Antenna}

A bi-conical antenna (or a bi-conical transmission line) is formed by placing two perfectly conducting cones tip to tip with their axis in opposite directions. Bi-conical antennas can also be formed by placing a perfectly conducting cone over a perfectly conducting ground as shown in Figure 7. In this case, the cone and its image through the ground plane form the bi-conical antenna. Since our analysis is conducted for a radiating system excited by a single current pulse we will use the latter in our analysis. An application of a voltage pulse across the input terminals at $\mathrm{O}$ will give rise to a current pulse that propagates along the surface of the cone with the speed of light, $c$. The current density associated with the current pulse is spread uniformly around the surface of the cone. This will give rise to a radiation field in the space located between the surface of the antenna and the ground. 


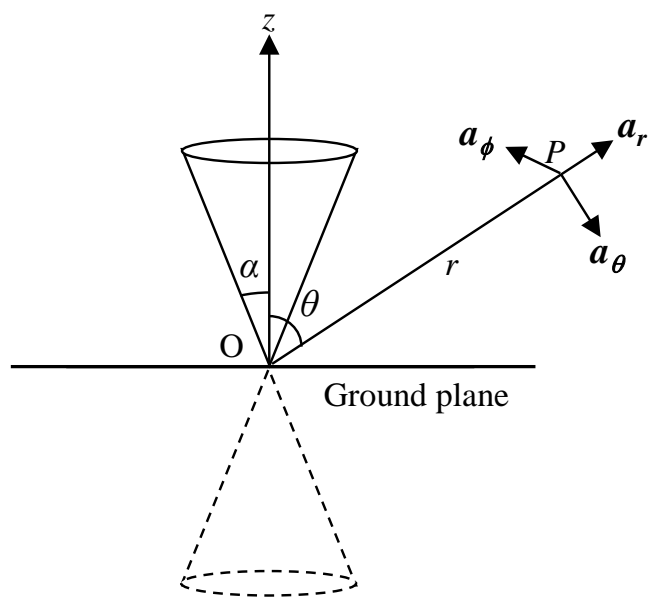

Figure 7. Bi-conical antenna with a semi cone angle of $\alpha$. The unit vector $\boldsymbol{a}_{\phi}$ is directed along the vector $\boldsymbol{a}_{r} \times \boldsymbol{a}_{\theta}$.

\subsubsection{Electromagnetic Fields of a Bi-Conical Antenna}

Expressions for the electromagnetic fields generated by a bi-conical antenna are available in many text books dealing with antenna theory [9]. According to these expressions the electric field at point $P$ (see Figure 7) generated by a bi-conical antenna with half apex angle $\alpha$ with a current $i(t)$ flowing from the origin is given for $t<l / c$ (i.e. the current has not reached the end of the antenna of length l) by

$$
\boldsymbol{E}_{\theta}=\frac{i(t-r / c)}{2 \pi r \varepsilon_{0} c \sin \theta} \boldsymbol{a}_{\theta}, \quad \theta>\alpha
$$

The magnetic flux density (or the B-field) associated with the radiation is directed along $\boldsymbol{a}_{\phi}$ and has the magnitude $E_{\theta} / c$. The Poynting vector associated with this electromagnetic field at point $P$ is given by

$$
\boldsymbol{S}=\frac{[i(t-r / c)]^{2}}{(2 \pi r)^{2} c \varepsilon_{0} \sin ^{2} \theta} \boldsymbol{a}_{r}
$$

Note that in writing down the above equation we have assumed that $\tau<l / c$. The total power radiated away at any given time $t$ is obtained by integrating the Poynting vector over a semi-spherical region of radius $r$ excluding the region of the antenna. The result of the exercise will be

$$
P(t)=\frac{[i(t-r / c)]^{2}}{(2 \pi)^{2} \varepsilon_{0} c} \int_{0}^{2 \pi} \int_{\alpha}^{\pi / 2} \frac{\mathrm{d} \theta}{\sin \theta}
$$

This spatial integral can be evaluated analytically and the result is

$$
P(t)=\frac{[i(t-r / c)]^{2}}{2 \pi \varepsilon_{0} c} \ln \left\{\cot \frac{\alpha}{2}\right\}
$$

\subsubsection{Energy Dissipation and the Action Associated with the Radiation of the Bi-Conical Antenna}

The total energy radiated by the current pulse as electromagnetic radiation can 
be obtained by integrating the expression obtained for the power over time and the result is

$$
U=\frac{q^{2}}{4 \pi^{3 / 2} \varepsilon_{0} c \sigma} \ln \left\{\cot \frac{\alpha}{2}\right\}
$$

The action associated with the radiation field is given by

$$
A=\frac{\tau q^{2}}{4 \pi^{3 / 2} \varepsilon_{0} c \sigma} \ln \left\{\cot \frac{\alpha}{2}\right\}
$$

Substituting for $\tau$ from Equation (3) we obtain

$$
A=\frac{\sqrt{2} q^{2}}{4 \pi \varepsilon_{0} c} \ln \left\{\cot \frac{\alpha}{2}\right\}
$$

Observe from Equation (40) that the action associated with a given charge increases with decreasing value of the semi-conical angle. Equation (40) can be written as

$$
A=\frac{\sqrt{2} q^{2}}{4 \pi \varepsilon_{0} c} \ln \left\{\frac{2 l}{a}\right\}
$$

In the above equation $l$ is the length of the bi-conical antenna and $a$ is the radius of the antenna at its end. Observe that the action increases with increasing $l$ and it decreases with increasing $a$. The maximum action is realized when $l$ has its maximum value and when $a$ has its minimum value. Considering, as previously, that the largest length of the antenna that can be realized in nature is the radius of the universe and the smallest value of $a$ is on the order of $a_{0}$, the maximum action corresponding to a given charge $q$ is given by

$$
A_{m}=\frac{\sqrt{2} q^{2}}{4 \pi \varepsilon_{0} c} \ln \left\{\frac{2 R_{u}}{a_{0}}\right\}
$$

This action (given in units of $h / 2 \pi$ ) as a function of charge $q$ (given in units of electronic charge) is shown in Figure 8. In making this diagram we have taken $a_{0}=5.29 \times 10^{-11} \mathrm{~m}$ and $R_{u}=4.4 \times 10^{26} \mathrm{~m}$. Observe that as the charge reduces to the electronic charge the maximum action reduces to a value on the order of $h / 2 \pi$. This shows that, within the confines of the natural limits, the maximum action that the radiation field of a bi-conical antenna excited by an electronic charge is about $h / 2 \pi$. If the charge is reduced below this the action will also be reduced below $h / 2 \pi$. We can summarize this result again using the inequality $A \geq h / 2 \pi \rightarrow q \geq e$. It is important to point out that in the above analysis we have tacitly assumed that, since the antenna is tapering off towards the ground, the radius of the conductor at sections below the upper end is less than $a_{0}$. However, in nature the limit of the radius that one can have is the Bohr radius. In practical applications the radius has to be larger and the length has to be smaller than the values used in estimating the above limiting value. But, a larger radius and a smaller length will not disturb the inequality derived here because they will give rise to a reduction in the action and the result being that much larger charges are needed to satisfy the condition $A>h / 2 \pi$, thus keeping the inequality still valid. 


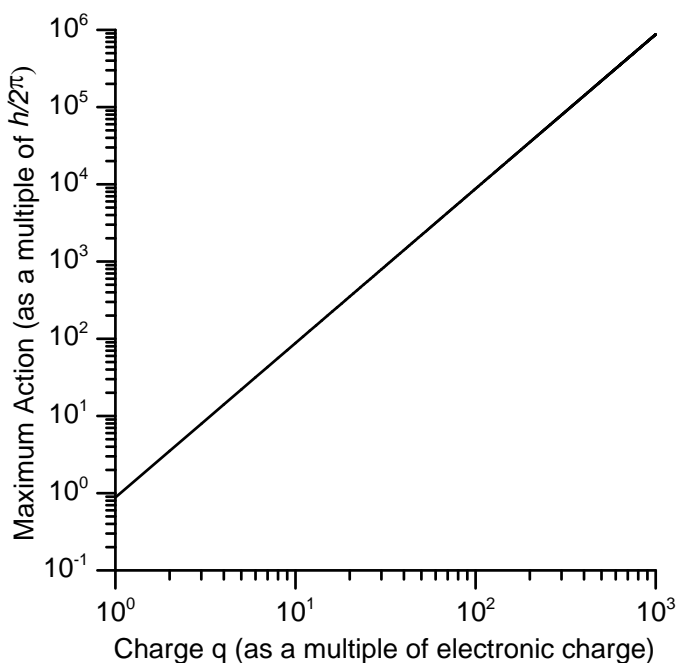

Figure 8. The maximum action (in multiples of $h / 2 \pi$ ) as a function of the charge $q$ (in multiples of electronic charge) pertinent to a bi-conical antenna. Note that as $q$ reaches the electronic charge the maximum action reaches about $h / 2 \pi$.

\section{Discussion}

In the previous sections, we have analyzed the radiation fields generated by four types of radiators, namely, short electric dipole, small magnetic dipole, travelling wave and bi-conical. The final conclusion we have reached after studying the action associated with the radiation fields generated by these radiators is the same. Of course, in the analysis we have assumed ideal conditions by neglecting losses associated with the attenuation and dispersion of the current as it travels along the radiator. But, as pointed out before, the final conclusion, namely, $A>h / 2 \pi \rightarrow q>e$, would not change if the losses are taken into account. It is important to stress here that the above inequality is an order of magnitude relationship and the left hand side of the inequality may vary between $A>h / 4 \pi$ and $A>h / 2 \pi$ from one case to another.

In the analysis presented here, the current is represented by a Gaussian pulse. We have repeated the analysis also using other symmetrical current waveforms such as finite step function and half sinusoidal function. All these waveforms generated results which are within $10 \%$ of each other and, therefore, do not disturb the order of magnitude inequality presented in this paper.

It is also important to point out that in the radiating systems analyzed here what is propagating along the radiator with the speed of light is the current waveform and not individual charges. One might wonder whether the final conclusion reached here would still remain valid if the radiation fields are generated by the acceleration of free electrons. Such an analysis was carried out by Cooray and Cooray [6]. In that work they have studied the radiation generated by the deceleration of uniformly moving electrons when they strike a perfectly conducting solid boundary. The study confirmed that the above inequality is satisfied also by the radiation fields generated by acceleration or deceleration of free 
electrons. That result, when combined with the results presented here shows that the above inequality is a condition that may be satisfied by electromagnetic radiation in general.

The above mentioned general result does not prove that electron is the smallest free charge that one can find in nature. If we have to prove that electron indeed is the smallest free charge available in nature then we have to show that $A \geq h / 2 \pi$ (or $A \geq h / 4 \pi$ ) is a law of nature. Fortunately, such a law exists in nature and it is called the time-energy uncertainty principle [10]. Cooray and Cooray [4] showed that the inequality $A \geq h / 2 \pi$ is valid for electromagnetic radiation generated by travelling wave antennas. Indeed, the results given above can be combined with this qualitative law to obtain an expression for the elementary charge. Details of this exercise are described in references [3] [4] [5] [6].

\section{Conclusion}

The results presented in this paper show that the action generated by the radiation fields of short electrical dipoles, small magnetic dipoles, travelling wave antennas and bi-conical antennas satisfy the order of magnitude inequality $A \geq h / 2 \pi \rightarrow q \geq e$ where $A$ is the action (product of energy radiated and the duration of emission), $h$ is the Planck constant, $q$ is the charge associated with the current that gave rise to the radiation and $e$ is the elementary charge. The results obtained here, in combination with the results obtained elsewhere, show that this inequality may be valid in general for the radiation fields as predicted by classical electrodynamics.

\section{Acknowledgements}

Authors appreciate the suggestions, support and encouragement given by Prof. Farhad Rachidi, Prof. Marcos Rubinstein, Prof. Carlo Mazzetti and Prof. Yoshihiro Baba during the development of ideas presented in this paper.

\section{References}

[1] Uman, M.A. and McLain, D.K. (1969), Magnetic Field of Lightning Return Stroke. Journal of Geophysical Research, 74, 6899-6910. https://doi.org/10.1029/JC074i028p06899

[2] Thottappillil, R., Schoene, J. and Uman, M.A. (2001) Return Stroke Transmission Line Model for Return Stroke Speed Near and Equal That of Light. Journal of Geophysical Research, 28, 3593-3596. https://doi.org/10.1029/2001GL013029

[3] Cooray, V. and Cooray, G. (2016) On the Remarkable Features of the Lower Limits of Charge and the Radiated Energy of Antennas as Predicted by Classical Electrodynamics. Atmosphere, 7, 64. https://doi.org/10.3390/atmos7050064

[4] Cooray, V. and Cooray, G. (2016) On the Momentum Transported by the Radiation Field of a Long Transient Dipole and Time Energy Uncertainty Principle. Atmosphere, 7, 151. https://doi.org/10.3390/atmos7110151

[5] Cooray, V. and Cooray, G. (2017) On the Action of the Radiation Field Generated by a Traveling-Wave Element and Its Connection to the Time Energy Uncertainty 
Principle, Elementary Charge and the Fine Structure Constant. Atmosphere, 8, 46. https://doi.org/10.3390/atmos8030046

[6] Cooray, V. and Cooray, G. (2017) Classical Electromagnetic Fields of Moving Charges as a Vehicle to Probe the Connection between the Elementary Charge and Heisenberg's Uncertainty Principle. Natural Science, 9, 219-230.

https://doi.org/10.4236/ns.2017.97022

[7] Pannofsky, W.K.H. and Phillips, M. (1962) Classical Electricity and Magnetism. Addison-Wesley, Reading.

[8] Smith, G.S. (1997) An Introduction to Classical Electromagnetic Radiation. Cambridge University Press, Cambridge, UK.

[9] Balanis, C.A. (1982) Antenna Theory: Analysis and Design. Harper and Row Publishers, New York.

[10] Wichmann, E.H. (1967) Quantum Physics (Berkeley Physics Course, Volume 4). McGraw Hill, New York. 